\title{
Design of multi-function remote control car
}

\author{
Shoucheng Zhao, Kun Zhang, Yibo Lu
}

School of Automotive Engineering, Chengde University of Technology, Hebei, China

\section{ABSTRACT}

With the rapid progress of computer, microelectronics and information technology, the development of intelligent technology is getting faster and faster, higher and higher, and the application scope has been greatly expanded. Intelligent as a modern new invention, is the future direction of development, it can be in accordance with the preset mode in an environment in the automatic operation, does not need man-made management, can be applied to scientific exploration and other purposes. Intelligent electric car is one of the embodiments. Designers can program their software to achieve their travel, tracking, stop the precise control and detection of data storage, display, without human intervention. Therefore, the intelligent electric car with reprogramming characteristics, is a kind of robot.

The design uses AT89C51 single-chip motor drive circuit and infrared remote control and tracking module and infrared receiver integrated sensor designed, the use of modular design, the use of infrared remote-control car forward, back, turn left, turn right, start and stop.

Keywords: single-chip, infrared remote control, DC power supply

Received: $18^{\text {th }}$ Jan. $2019 \quad$ Accepted: $6^{\text {th }}$ Apr. 2019 Online: $20^{\text {th }}$ Apr. 2019

\section{Introduction}

With the continuous development of the distance education system and the increasingly perfect, the use of multi-media as a means of teaching at all levels of schools have been widely used. In recent years, the use of multimedia teaching system and development, often encountered simultaneously using a variety of devices, such as: digital projectors, DVD, $\mathrm{VCD}$, video recorders, televisions, due to various devices that are equipped with remote control, and different equipment to follow the infrared transmission protocol is not the same, the operation of these devices have to use a variety of remote control, to the user has brought a lot of inconvenience. The theme of this graduation design is infrared remote-control circuit design. Infrared remote control is characterized by the use of infrared for point-to-point communication technology, does not affect the surrounding environment, do not interfere with other electrical equipment. Indoor distance (less than 10 meters), the signal without interference, high transmission accuracy, small size, low power characteristics, remote control has been widely used. Through the MCU-based control instructions to a variety of devices for remote control, you can choose a different button to control different devices. Thus convenient and quick to achieve remote control.

Commonly used infrared remote-control system is generally divided into two parts. The main components of the transmitting section are infrared light emitting diodes. It is actually a special light-emitting diode; because of its internal material is different from ordinary light-emitting diodes, so at both ends of the application of a certain voltage, it is issued by the infrared rather than visible light. Infrared light-emitting diodes are generally black, dark blue, transparent three colors.

The receiving part of the infrared receiver tube is a photodiode. In practical applications to the infrared receiver diode to reverse bias, it can work properly, that is, infrared receiver diode in the circuit is used in reverse use, so as to get a higher sensitivity. Infrared light-emitting diodes are generally round and square too. As the infrared light emitting diode transmission power is generally small, so the infrared receiver diode receives the signal is relatively weak, so it is necessary to increase the high gain amplifier circuit. In recent years, whether it is amateur production or formal products, most of them are complete infrared receiver.

Copyright (C) 2019 Zhao et al. This is an Open Access article distributed under the terms of the Creative Commons Attribution-NonCommercial 4.0 International License (http://creativecommons.org/licenses/by-nc/4.0/), permitting all non-commercial use, distribution, and reproduction in any medium, provided the original work is properly cited. 


\section{Design requirements and indicators}

Infrared remote control is currently using more of a remote control means. Infrared remote-control device has a small size, low power consumption, strong function, low cost. In the family life, tape recorders, audio equipment, air conditioning color TV are used infrared remote-control system. The design requires the use of infrared transmission control instructions and intelligent control system, with a powerful and flexible control of the microprocessor to issue pulse coding, composed of a remote control system. Infrared coding is the data transmission quality is a pulse width modulation serial communication. Infrared communication part of the transmission is mainly to send the data into a certain format of the pulse, and then drive the infrared LED to send data out. The receiving part is the completion of the infrared reception, amplification, demodulation, reduced to synchronous transmission format the same, but the high and low potential just the opposite of the pulse signal, the main output TTL compatible level. And finally through the decoding of the pulse signal into data, in order to achieve data transmission. The main technical indicators of the design are as follows:

(1) Remote control range: 4-6 meters

(2) Shows a controllable channel

(3) To receive sensitive and reliable, anti-interference ability

(4) Control electrical current up to $2 \mathrm{~A}$.

\subsection{Design of Infrared Remote-Control System}

Infrared remote-control system consists of two parts of the transmitter and receiver, the system uses coding / decoding ASIC and microcontroller chip to control the operation. The design of the circuit consists of the following basic modules: DC power supply, infrared transmitter circuit, infrared receiver circuit and control part. The system block diagram is shown in Figure 1.

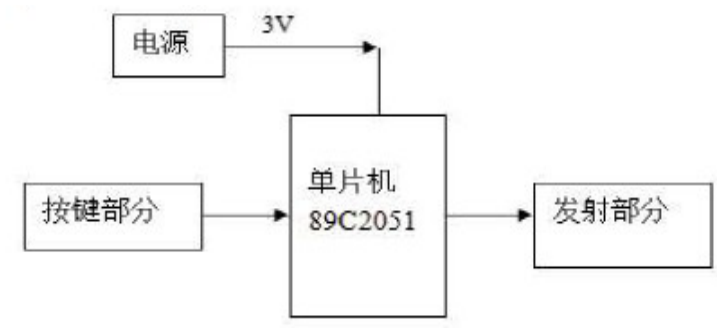

图 2-1 红外谣控电路啀图（a）发射电路枉图

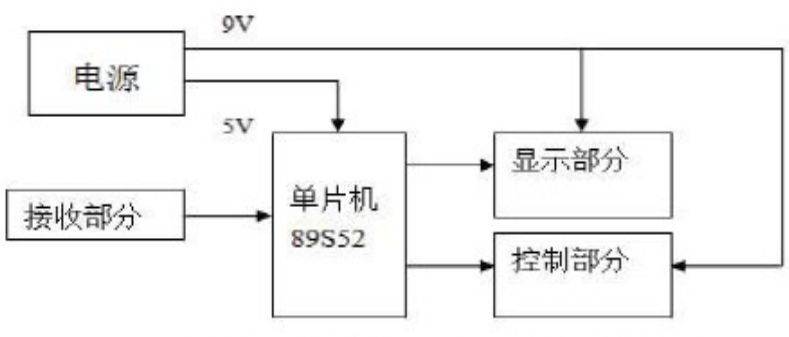

图 2-1 红外遊控电路框图（b）接收电路枉图

Figure 1. Infrared remote control circuit block diagram (a) transmission circuit block diagram

Figure 2. Infrared remote control circuit block diagram (b) block diagram of the receiving circuit

\section{Infrared transceiver circuit design}

\subsection{Main chip-lightning storage type microcontroller AT89S52 introduction}

\subsubsection{The AT89S52 has the following main features:}

(1) $8 \mathrm{~KB}$ can be adapted to program Flash memory (Can withstand 1,000 write / erase cycles)

(2) Three-level program memory is confidential

(3) $256 * 8$ bytes of internal RAM 
(4) 32 programmable I / O lines

(5) 3 16-bit timers / counters

(6) 6 interrupt sources

(7) Programmable serial channel

(8) On-chip clock oscillator

AT89S52 is designed with static logic, and provides two available software to choose the power-saving mode - idle mode and power-down mode. In the idle mode, the CPU stops working, and RAM, timer / counter, serial port and interrupt system continue to work. In the power-down mode, the on-chip oscillator is stopped and the clock is 'frozen', all functions are paused, only the contents of the on-chip RAM are saved until the next hardware reset.

\subsubsection{AT89S52 pin}

89S52 single-chip pin description as shown:

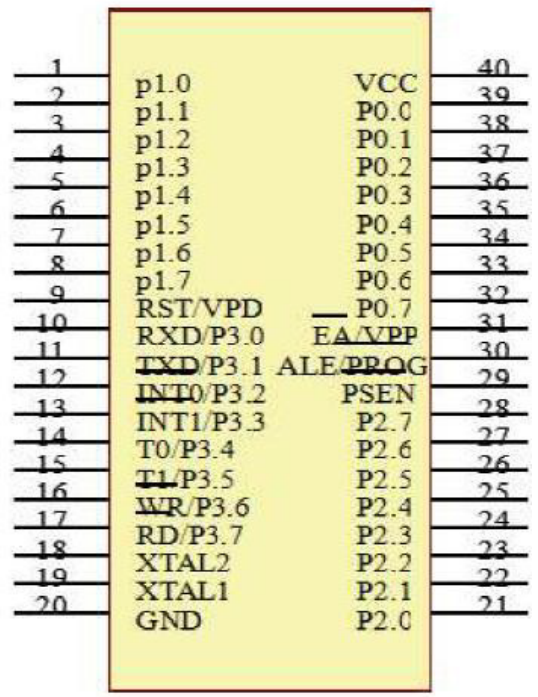

Figure 3.

\subsection{Introduction to $89 \mathrm{C} 2051$}

89C2051 has 20 pins, as shown in Figure 3. P1 port total consists of 8 feet and quasi-two-way port. P3.0-P3.6 7-pin, quasi-bidirectional port, such as P3.0, P3.1 serial communication function, P3.2, P3.3 interrupt input function, P3.4, P3.5 of the timer input function.

In the pin drive capability, 89C2051 has a strong pull-down capability, P1, P3 port pull-down capability can reach $20 \mathrm{~mA}$. In contrast, 89C51 port pull-down capacity of up to $15 \mathrm{~mA}$ per foot. But the sum of the 9-pin current is less than $71 \mathrm{~mA}$. Thus, the average current of the pin is only 9mA. 89C2051 drive capacity enhancement, making it can directly drive LED digital tube.

Compared to the 89C51 it has a number of features, but it is less power, easy to carry, more economical in the launch circuit plays an important role. Therefore, in the design of the infrared emission circuit to use it to achieve the pulse signal generation. 


\begin{tabular}{|c|c|c|c|}
\hline & U1 & $89 \times 205$ & \\
\hline 1 & \multirow{3}{*}{$\begin{array}{l}\text { RST } \\
\text { (RXD)P3 }\end{array}$} & \multirow{2}{*}{ VCC } & 20 \\
\hline 2 & & & 19 \\
\hline 3 & & & 18 \\
\hline 4 & (IXD) & $\begin{array}{l}11.0 \\
D 1.5\end{array}$ & 17 \\
\hline$\underline{5}$ & YIALL & $\begin{array}{l}\text { P1.3 } \\
\text { D114 }\end{array}$ & 16 \\
\hline 6 & $\frac{\text { XIAL1 }}{\text { (INTR) D 3 } 2}$ & $\begin{array}{l}\text { P1.4 } \\
\text { P1.3 }\end{array}$ & 15 \\
\hline 7 & 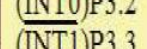 & $\begin{array}{l}1.3 \\
\mathrm{P} 12\end{array}$ & 14 \\
\hline 8 & (TOP 34 & D1 $1(4 \mathrm{~N} 1 \mathrm{2}$ & 13 \\
\hline 9 & (10) $1 \mathrm{D}_{3} 4$ & P1 (AINI) & 12 \\
\hline 10 & GND & 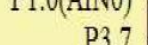 & 11 \\
\hline
\end{tabular}

Figure 4. Pin of $89 \mathrm{C} 2051$

\subsection{System function implementation method}

\subsubsection{Encoding format of remote-control code}

The remote control using the number of pulses, different pulse number represents a different code, the minimum for the two pulses, the maximum of 17 pulses. In order to make the reception reliable, the first bit code width of $3 \mathrm{~ms}$, the remaining $1 \mathrm{~ms}$, remote control code data frame interval is greater than 10ms, as shown in Figure 3-3.

\subsubsection{Launch of remote-control code}

Using the 89C2051 chip. With the P1 port composed of keyboard, access to key, with the internal timer 1 to produce a $40 \mathrm{KHz}$ software interrupt, as the infrared modulation of the fundamental, when an operation button press, the microcontroller first read the key value, and then the key value set the number of remote control code pulse, and then modulated into $40 \mathrm{kHz}$ square wave emitted by the infrared tube. The output modulation waves of port P3.5 is shown in Figure 5 .

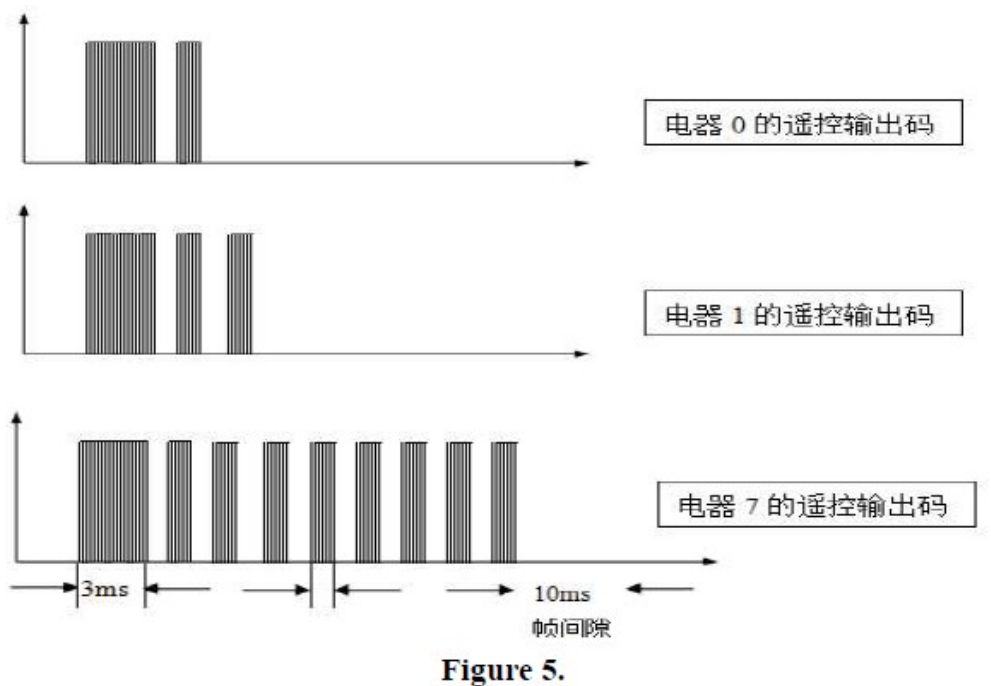

\subsection{Infrared emission circuit}

Remote control transmission through the keyboard, each press a key, that is generated with different encoded digital pulse, this code command signal modulation in the $40 \mathrm{KHz}$ carrier, the excitation infrared light diodes produce different pulses, through the space to the controlled machine remote control receiver. P1 port as a key part, P3.5 port as the launch part, and then use the transistor to drive the infrared emission. The circuit is shown in Figure 6. 


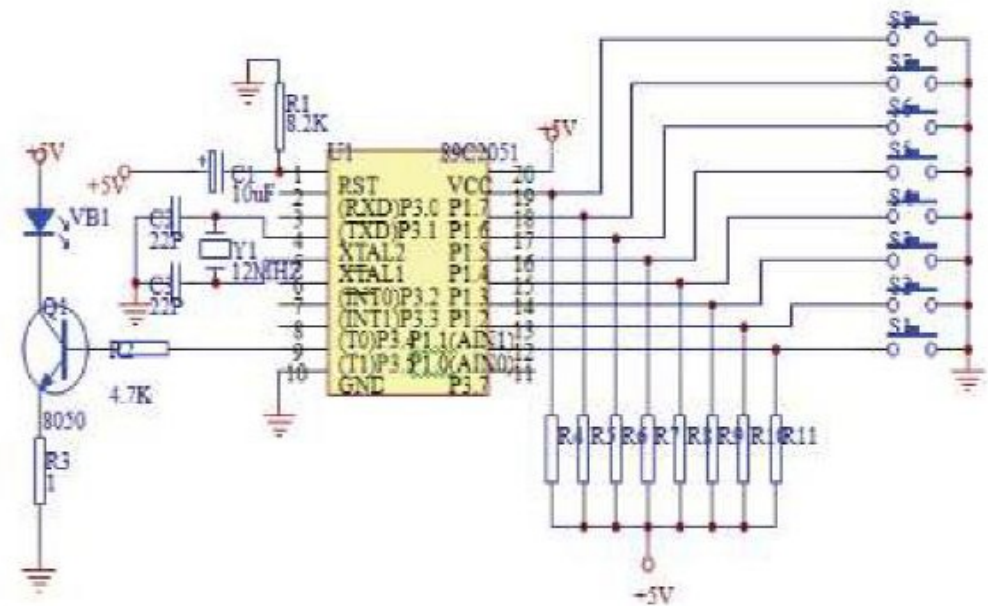

Figure 6. Infrared emission circuit

\subsection{Infrared receiver circuit}

In the process of receiving, the pulse through the optical filter and infrared diode is converted to $40 \mathrm{KHz}$ electrical signal, this signal through the amplification, detection, shaping, demodulation, sent to the decoding and interface circuit, thus completing the corresponding remote control function. The receiving circuit is shown in Figure 7.

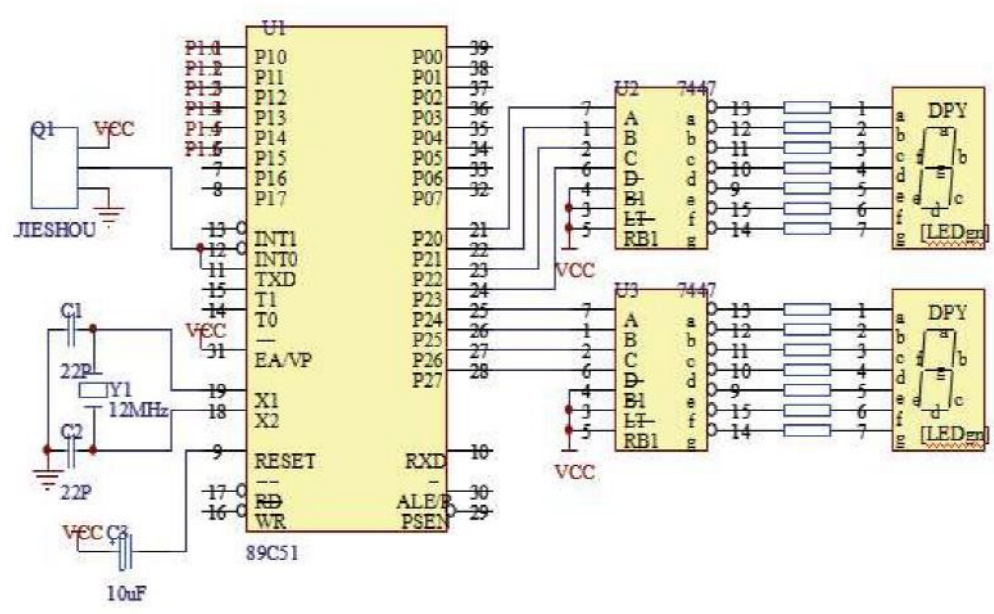

Figure 7. Receive circuit diagram

Usually, the infrared remote control will remote control signal (binary pulse code) modulation in the $40 \mathrm{KHz}$ carrier, the buffer amplified and sent to the infrared light-emitting diodes, resulting in infrared signal emission. The abovementioned remote control pulse to the frequency of $40 \mathrm{kHz}$ (cycle of $26.3 \mathrm{~ms}$ ) carrier signal pulse amplitude modulation (PAM), and then amplified by the buffer to the infrared LED, the remote-control signal emitted.

According to the remote control signal coding and transmission process, the remote control signal identification - that is, the decoding process is to remove the $40 \mathrm{kHz}$ carrier signal to identify the binary pulse code 0 and 1 . By the MCS-51 series microcontroller AT89S52, integrated infrared receiver, memory, reduction modulation and infrared LED drive circuit components.

\subsection{Software design}

\subsubsection{Emission coding software design}

First, the timer is initialized to a time period with a frequency of $40 \mathrm{kHz}$. When a button is pressed, data 1 is sent to start working. At the same time the timer overflow, that is, the timer is full, the implementation of timer interrupt, interrupt procedures are as follows: 
INTT1: CPL P3.5; 40KHZ infrared remote-control signal generation

RETI: interrupt return

Thus generating a carrier signal of $40 \mathrm{kHz}$.

When sending data 0 , the timer does not work.

Send the program as follows:

REMOTE: MOV R1, A; the number of incoming pulses

LJMP OUT3; turn the first code to send processing

OUT: MOV R0, \# 55H; 1MS wide low-level emission control data

OUT1: SETB ET1; ON T1 interrupt

SETB TR1; Turns on timer T1

NOP; delay

NOP

NOP

NOP

NOP

DJNZ R0, OUT1; time not to turn OUT1 to recycle

MOV R0, \# 32H; 1MS high level gap control data

OUT2: CLR TR1; OFF timer T1

CLR ET1; OFF T1 interrupt

CLR P3.5; off pulse output

NOP; empty operation delay

NOP

NOP

NOP

NOP

NOP

NOP

NOP

NOP

NOP

NOP

DJNZ R0, OUT2; time not to turn OUT2 to recycle

DJNZ R1, OUT; pulse is not fi nished, turn the OUT recirculation launch

LCALL DL500MS;

RET;

OUT3: MOV R0, \# 0FFH; loaded with 3MS wide control data

LJMP OUT1; turn OUT1

\subsubsection{Receiving Encoded Software Design}

Single-chip power-on reset, the first of its internal timer initialization, timer and software count method, when there is a signal input, the microcontroller interrupt, and P3.1 port count pulse number, measured P3.1 high, the width of the low level. P3.1 pin is usually high, when receiving the infrared remote-control signal, due to the integration of the infrared receiver head reverse role, INT0 pin jump to low, calculate the number of pulses through the 7447-decoding circuit, the digital tube shows the corresponding value. 
The following is the first $3 \mathrm{~ms}$ pulse decoding program:

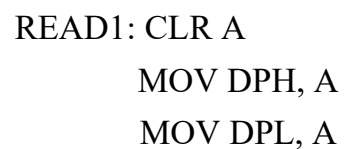

HARD1: JB P3.1, HARD11

INC DPTR NOP

NOP

AJMP HARD1

HARD11: MOV A, DPH

JZ READOUTT0

CLR A

READ11: INC A

READ12: JNB P3.1, READ12

MOV R1, \# 06H

READ13: JNB P3.1, READ11

LCALL DELAYREAD

DJNZ R1, READ13

\section{DC power supply design}

\subsection{DC power supply using single-phase rectifier circuit rectifier}

The rectifier circuit mainly converts the alternating current into direct current. To achieve this goal is mainly relying on the diode one-way conductive effect, so the diode is a key component of the rectifier circuit. I use a single bridge rectifier circuit. The design of the rectifier circuit shown in Figure 8.

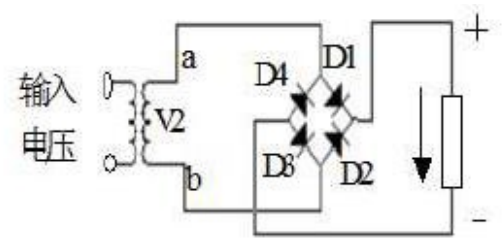

Figure 8. single bridge rectifier circuit diagram

\section{2. $\quad$ Filter circuit}

In the rectifier circuit output waveform because it contains more ripple components, and the required waveform is not consistent. So the rectifier circuit after the filter circuit to filter out the rectified output voltage ripple. The filter circuit often capacitor filter, inductor filter and RC filter. The circuit uses a capacitor filter circuit. As shown in Figure 9.

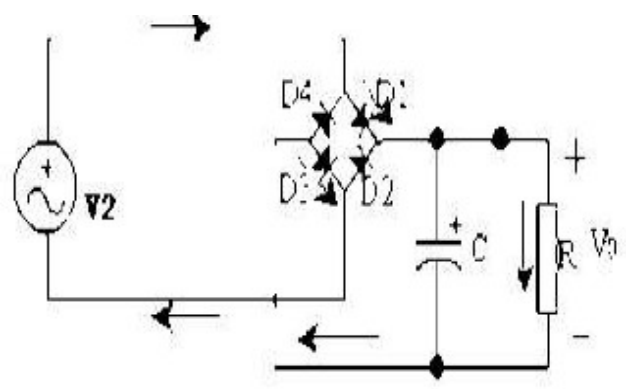

Figure 9. Capacitance filter circuit 


\subsection{Voltage regulator circuit}

Typical application circuit shown in Figure 4-3. Figure C1, C2 for frequency compensation to prevent self-oscillation and inhibit high-frequency interference; $\mathrm{C} 3$ using electrolytic capacitors to reduce the power supply to introduce the impact of low-frequency interference on the output voltage; $\mathrm{D}$ is the protection of the diode, when the input short-C 3 a discharge path to prevent the voltage across the $\mathrm{C} 3$ push through the tube to send the junction.

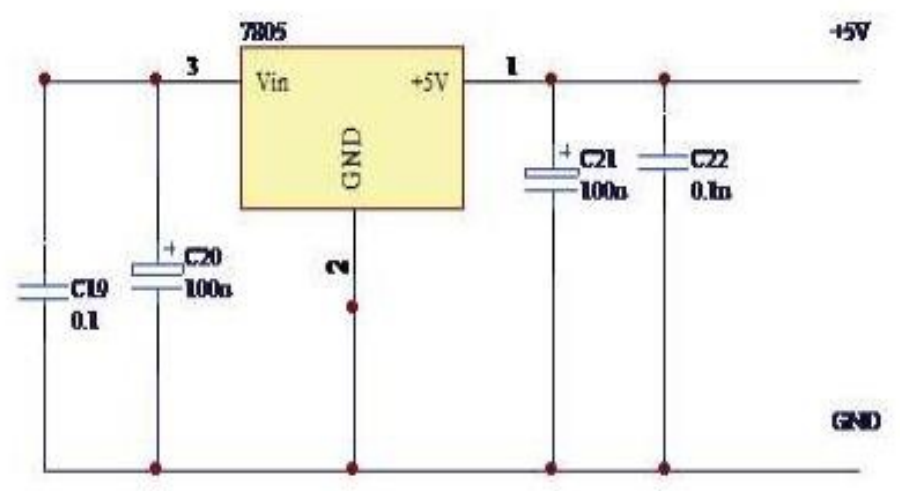

Figure 10. Regulator circuit diagram

\section{Control part}

In the control part of the isolation drive circuit, with optoelectronic devices as isolation components, the use of optocouplers to isolate strong electricity to prevent the strong impact of the work of the microcontroller. Optocoupler is a combination of light-emitting diodes and phototransistor devices, light-emitting diode is the input side of the electrical signal into the same regular changes in light, and the photosensitive transistor is the light again into the same changes in the law of the electrical signal, so the light plays the role of the medium. As the optocoupler anti-interference ability, it is easy to complete the level of matching and transfer, and not by the signal source is grounded. So the application is increasingly widespread.

The purpose of optoelectronic isolation is to cut off the electrical connection between the two circuits, so that they are independent at each other, and thus cut off the noise from one circuit into another circuit path. Photoelectric isolation is achieved by optocoupler. The shell has two kinds of metal or plastic. The light-emitting diodes and the phototransistors are filled with transparent insulators and align luminous tubes with the photoresist to increase their sensitivity. The circuit symbols for the opt couplers are shown in Figure 11.

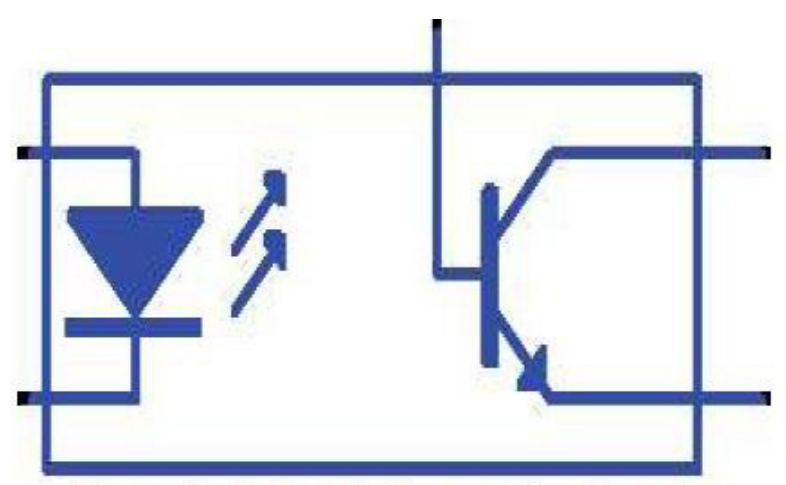

Figure 11. Schematic diagram of optocoupler

The coupling response speed and sensitivity can be controlled by the grounding resistance. In general, the smaller the resistance, the higher the response speed. The circuit is shown in Figure 12. 


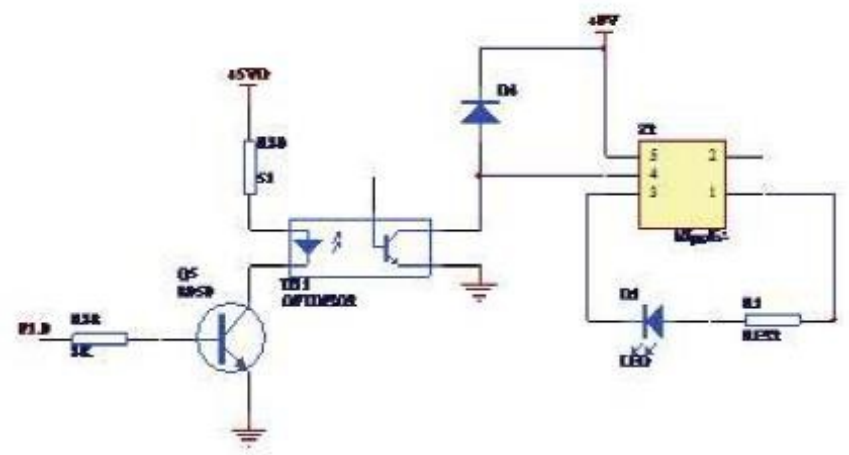

Figure 12. Control circuit diagram

Through the optocoupler, the use of relays can be achieved on different devices or other equipment to be controlled, so as to achieve a weak power, and to control the strong power function, but also to control different devices. Here to replace the light-emitting diodes, in fact, can control a number of different strong electrical equipment.

\subsection{Motor drive module}

The module mainly by the chip L298 control two motor forward and reverse, and change the motor speed, the circuit shown in the figure. The L298 chip is a high voltage, high current dual full bridge driver. Which SENSEA, SENSEB, respectively, for the two $\mathrm{H}$ bridge current feedback feet, when not directly grounded. VCC, VS is connected to the power supply pin, the voltage range is $4.5 \sim 7 \mathrm{~V}, 2.5 \sim 46 \mathrm{~V}$, the design of the VCC side and the microcontroller power supply side of the common $5 \mathrm{~V}$ power supply, VS end independent of $9 \mathrm{~V}$ power supply. ENA, ENB is enabled, low level is disabled. IN1, IN2, IN3, IN4 for the data input pin, OUT1, OUT2, OUT3, OUT4 for the data output pin. D1 D8 is the protection diode (IN5819), used to release the motor stop generated when the response peak potential, otherwise it will damage L298.

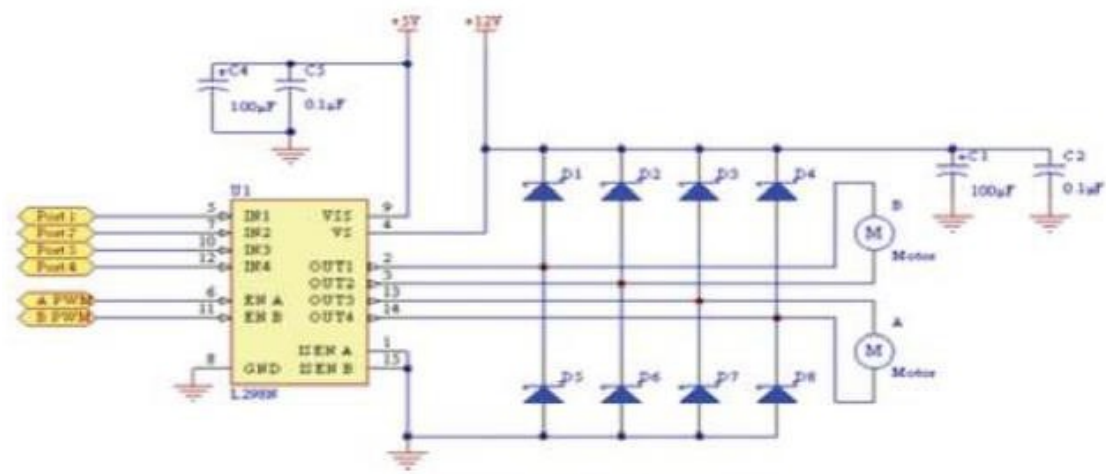

Figure 13.

\subsection{Obstacle Detection and Tracing Modules}

Obstacle detection and trajectory detection principle is the same. From the economic point of view, the module uses a reflective optocoupler, the circuit shown in Figure. Reflective optocoupler consists of an infrared emission tube and a phototransistor. LM324 is the voltage comparator, when the level of 3 feet is greater than 2 feet, the output pin 1 output high, and vice versa output low. High and low value depends on the LM324 2 feet level, adjust the potentiometer R23 LM324 2 pin voltage is $3 \mathrm{~V}$.

The obstacle avoidance circuit is installed on the left and right sides of the head of the car, for detecting obstructions of both sides. The working process is: when there is no obstacle, no infrared signal is reflected; photosensitive transistor cut off, LM324 3-feet in the R16 under the action of high-level (5V), greater than 2 feet (3V), the output high; When obstacles encountered, infrared reflected, photosensitive transistor conducts, the comparator 3 feet ground, less than 2 feet voltage (3V), the output is low. SCM according to the level of change to determine whether there are obstacles, when the left side of the obstacles encountered when the car turn right, when the right encounter obstacles when the car turns left. 
The tracking circuit is mounted on the left and right sides of the bottom of the car, and the traversing is done by discerning black and white. The working process is: infrared emission tube to send infrared light, when encountered black, does not reflect the infrared light, the comparator output is high; when encountered white line, red light reflected back, the comparator output is low. When the left side detects the white when the car turns right, when the right side of the white car when the car turned left, when the two sides are detected when the black car forward, when both sides are detected when the white car stopped.

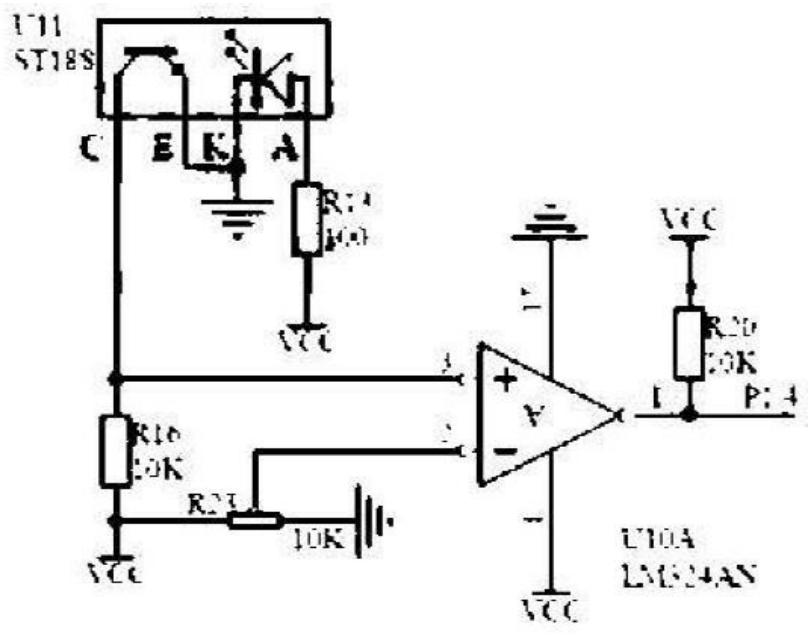

Figure 14.

\section{Debug results and their analysis}

The circuit designed a total of eight input keys, 7,8 for the special button.

When a key 5 is input, the device of the relay 5 is illuminated by the infrared transmitting and receiving circuit, and the number of the LEDs on the digital tube is displayed.

When the button 5 is pressed again, the No. 5 LED is off and the digital tube shows 0.

When the two keys 3 and 4 are pressed at the same time, the No. 3 and No. 4 diodes are bright and the digital tube displays 2 .

When the press button 7, all the equipment does not work, digital display 0 , light-emitting diodes are not light.

When the press button 8 , all the equipment is working, digital display 6 , light-emitting diodes are light. The design in the debugging process also encountered many problems.

(1) Circuit requires remote control distance of $4-6 \mathrm{~m}$, in the use of $38 \mathrm{KHz}$ receiver, although the signal can be received, but the distance is very limited. After repeated debugging, for the $40 \mathrm{KHz}$ receiver when the basic meet the design requirements.

(2) As the $3 \mathrm{~ms}$ receive pulse on the back of $1 \mathrm{~ms}$, coding and demodulation error, resulting in the receiver no signal output. The solution is to receive the signal by placing $3 \mathrm{~ms}$ of received pulse in front of it. Because the circuit in the decoding process, the microcontroller to receive digital frame processing, the first is $3 \mathrm{~ms}$ pulse test, when the first low-level code pulse width is less than $2 \mathrm{~ms}$ will be wrong.

In the initialization process, the $\mathrm{P} 1$ port is set to 0 , but the relay is still working, through repeated debugging, the initial $\mathrm{P} 1$ port set all 1, through the reverse make all the output 0 , to meet the power-on reset, the relay power, meet initialization requirements. 


\section{Conclusion}

As the current remote-control device for a single device and a separate control, together the design of the infrared remote-control circuit design of a number of control buttons, you can supply different devices, you can also use the same device for multiple functions of different control. Basically, meet the technical requirements.

But the circuit is also imperfect, it can only achieve a single channel control of multiple devices, that is, it cannot control two or more devices at the same time.

In the design process, through a lot of access to information, careful study of teaching materials, the micro-controller has a more profound understanding of the design software, carefully analyze the hardware circuit, draw the program flow chart, cultivate my patience and hard the spirit of study.

Through this graduation design so that I understand their original knowledge is too theoretical, and face a separate subject feel very at a loss. Don't know where to start, don't know how to achieve their own purpose to plan how to achieve the purpose of the process. I always feel that I knew it all, that I can do anything, but now it seems like I am too confident about it. Through this graduation design, I understand that learning is a long process of accumulation in the future work and life ought to continue to learn, and strive to improve their knowledge and comprehensive quality.

\section{References}

1. National College Students Electronic Design Competition Organizing Committee. Fifth National CollegeStudents Electronic Design Competition Award-winning works selected, 1st edition, Beijing Institute of Technology Press, 2005, P10-17.

2. HG. Kang, DC. Chen. Electronic technology basic simulation part, 4th edition, higher education press, 1999, fourth edition, P82-155.

3. HG. Kang, SB. Zou. Electronic technology based on the digital part, 4th edition, Higher Education Press, 2000, 4th edition, P83-155.

4. JC. Li, RM. Cai. Commonly used crystal diodes, high power transistor manual, People's Posts and Telecommunications Press, 1981, the 1st edition, P23-55.

5. ZW. Huang, Y. Wang, WG. Chen. National College Students Electronic Design Competition Training Course, 1st edition, Electronic Industry Press, 2005, P304-P314.

6. JX. Wu, QY. Shen, TJ. Guo .8051 single chip microcomputer practice and application, the fi rst edition, Tsinghua University Press, 2002, P147-167.

7. Aoki Yoshihiko. Design and production of analog circuits, 1st edition, Science Press, 2005, 119-131.

8. GD. Li, YX. Zhu, XS. Wang. Single-chip foundation, 2nd edition, Beijing University of Aeronautics and Astronautics Press, 2001, P13-71.

9. LF. Mei, YQ. Wang, J. Zhang, etc. Single-chip principle and interface technology, the fi rst edition, Tsinghua University Press, 2004, P296-323. 